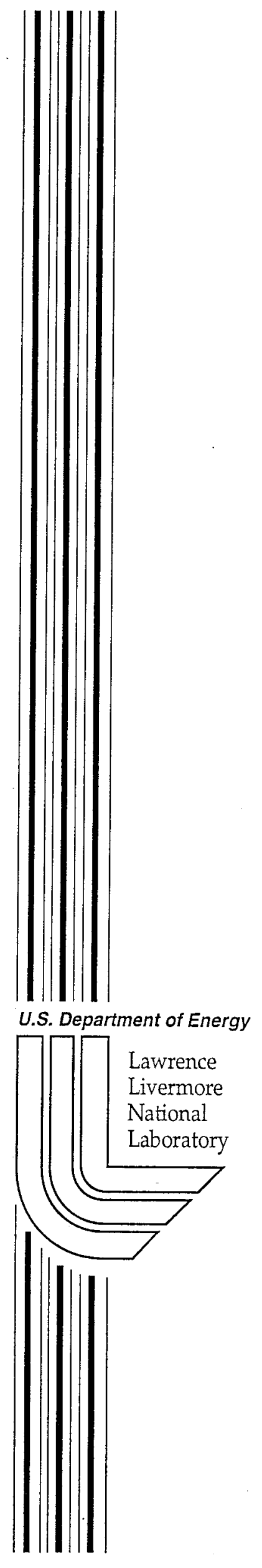

\title{
Electronic Effects on Grain Boundary Structure in BCC Metals
}

G.H. Campbell, W.E. King, J. Belak, J.A. Moriarty, S.M. Foiles

This article was submitted to

1999 Materials Research Society Fall Meeting, Boston, MA, November 29-December 3, 1999

\section{November 15, 1999}




\section{DISCLAIMER}

This document was prepared as an account of work sponsored by an agency of the United States Government. Neither the United States Government nor the University of California nor any of their employees, makes any warranty, express or implied, or assumes any legal liability or responsibility for the accuracy, completeness, or usefulness of any information, apparatus, product, or process

disclosed, or represents that its use would not infringe privately owned rights. Reference herein to any specific commercial product, process, or service by trade name, trademark, manufacturer, or otherwise, does not necessarily constitute or imply its endorsement, recommendation, or favoring by the United States Government or the University of California. The views and opinions of authors expressed herein do not necessarily state or reflect those of the United States Government or the University of California, and shall not be used for advertising or product endorsement purposes. 


\title{
ELECTRONIC EFFECTS ON GRAIN BOUNDARY STRUCTURE IN BCC METALS
}

\author{
GEOFFREY H. CAMPBELL, * WAYNE E. KING, * JAMES BELAK,** JOHN A. MORIARTY,** \\ AND STEPHEN M. FOILES ${ }^{\S}$ \\ * University of California, Lawrence Livermore National Laboratory, Chemistry and Matcrials \\ Science Directorate, P.O. Box 808, Livermore, CA 94550 \\ ** University of California, Lawrence Livermore National Laboratory, Physics Directorate, P.O. \\ Box 808, Livermore, CA 94550 \\ ${ }^{\S}$ Sandia National Laboratories, Livermore, CA 94550
}

\section{ABSTRACT}

The dominant factor in determining the atomic structure of grain boundaries is the crystal structure of the material, e.g. FCC vs. BCC. However, for a given crystal structure, the structure of grain boundaries can be influenced by electronic effects, i.e. by the element comprising the crystal. Understanding and modeling the influence of electronic structure on defect structures is a key ingredient for successful atomistic simulations of materials with more complicated crystal structures than FCC. We have found that grain boundary structure is a critical test for interatomic potentials. To that end, we have fabricated the identical $\sum 5(310) /[001]$ symmetric tilt grain boundary in three different $\mathrm{BCC}$ metals ( $\mathrm{Nb}, \mathrm{Mo}$, and $\mathrm{Ta}$ ) by diffusion bonding precisely oriented single crystals. The structure of these boundaries have been determined by high resolution transmission electron microscopy. The boundaries have been found to have different atomic structures. The structures of these boundaries have been modeled with atomistic simulations using interatomic potentials incorporating angularly dependent interactions, such as those developed within Model Generalized Pseudopotential Theory. The differing structures of these boundaries can be understood in terms of the strength of the angular dependence of the interatomic interaction. We report here the results for Ta.

\section{INTRODUCTION}

Atomistic simulations are an increasingly important means of understanding the behavior of materials under a variety of conditions. With this technique, an assembly of thousands, or even millions, of atoms is defined in a computer simulation and allowed to interact according to certain rules and boundary conditions. The boundary conditions include temperature and states of stress, allowing the calculation of such properties as the equation of state or unstable stacking fault energy. The structure and properties of crystal defects can also be predicted, such as the stressed configuration of a dislocation core or the interaction energy of an interstitial with a vacancy (see e.g. [1]). The rules of interaction are often very simple in order to speed computation. This simplification requires approximations to be made about the physics of the interacting atoms. Hence, in the development of models of interatomic interactions, an evaluation is necessary of whether the essential physics have been incorporated in the model. The models are tested by comparing their predictions with experimental observations.

Recently developed models of interatomic interactions incorporate angularly dependent contributions to model materials with directional bonding [2-7], such as the body centered cubic transition metals in which the $\mathrm{d}$ - bands participate in bonding. The strength of the directional component of the bonding has a major influence on the structure of crystal defects. The model of interatomic interactions with angular dependence that we use is the Model Generalized Psuedopotential Theory (MGPT) [5]. We have applied it to modeling the $\Sigma 5(310) /[001]$ symmetric tilt grain boundary (STGB) in niobium [8], molybdenum [9], and tantalum as a critical test of its accuracy. We report here on the results for Ta.

\section{ATOMISTIC SIMULATIONS}

For an elemental bulk transition metal, the generalized pseudopotential theory (GPT) [10] provides a rigorous real - space expansion of the total energy for a system of $\mathrm{N}$ ions in the form: 


$$
E_{t o t}\left(R_{1} \ldots R_{N}\right)=N E_{v o l}(\Omega)+\frac{1}{2} \sum_{i, j} v_{2}(i j, \Omega)+\frac{1}{6} \sum_{i, j, k} v_{3}(i j k, \Omega)+\frac{1}{24} \sum_{i, j, k, l} v_{4}(i j k l, \Omega),
$$

where $R_{i}$ denotes the position of ion $i, \mathrm{~W}$ is the atomic volume, and self interaction terms $(i=j=k=l$ ) are excluded from the summations. The leading volume term in the expansion, $E_{v o l}$, as well as the two -, three -, and four - ion interatomic potentials, $v_{2}, v_{3}$, and $v_{4}$, are volume dependent, but structure independent quantities and thus transferable to all bulk ion configurations, including all structural phases as well as the imperfect bulk solid with either point or extended defects present. The angular multi - ion potentials, $v_{3}$ and $v_{4}$, reflect contributions from partially filled $d$ - bands and are generally important for central transition metals. In the full GPT, however, these potentials are long - ranged, non - analytic, and multidimensional functions, so that $n_{3}$ and $n_{4}$ cannot be readily tabulated for application purposes. This has led to the development of a simplified model GPT or MGPT for BCC transition metals $[5,11]$. In the MGPT, the multi - ion potentials are systematically approximated by introducing canonical $d$ - bands and other simplifications to achieve short - ranged, analytic forms, which can then be applied to both static and dynamic simulations. To compensate for the approximations introduced, a limited amount of parameterization is allowed in which the coefficients of the modeled potential contributions are constrained by external experimental or $a b$ - initio theoretical data. Useful MGPT potentials have been so determined for Ta over a wide volume range.(ref.)

An ideal $\Sigma 5$ (310)/[001] STGB was constructed in the computer using the rules of the CSL theory [12]. To accommodate the interaction range of the MGPT potentials, the grain boundary plane contained two [ $1 \overline{3} 0]$ repeat lengths and six [001] repeat lengths. Two [310] repeat lengths were used on each side of the grain boundary resulting in a simulation cell containing $\mathrm{N}=960$ atoms. Convergence of the calculation was checked by doubling the simulation cell along the grain boundary normal to $\mathrm{N}=1920$ atoms. No additional significant relaxation was found and all results reported here employ the $\mathrm{N}=960$ atom cell. Periodic boundary conditions were applied within the grain boundary plane and free surface boundaries were applied along the grain boundary normal. However, all atoms within the MGPT potential interaction range of the free surface were fixed at the bulk lattice positions. These fixed atoms also serve as handles to position the two grains relative to one another. The system was brought to the position of minimum energy using a standard molecular dynamics computer code and the method of simulated annealing [13]. To explore the energy dependence of the state of relative translation between the crystals, one crystal was systematically shifted with respect to the other crystal along [1 $\overline{3} 0]$, the structure was relaxed in two dimensions, and the excess energy of the boundary calculated. At the minimum energy position for this process, a full three dimensional relaxation was performed. The results are shown in Fig.1.

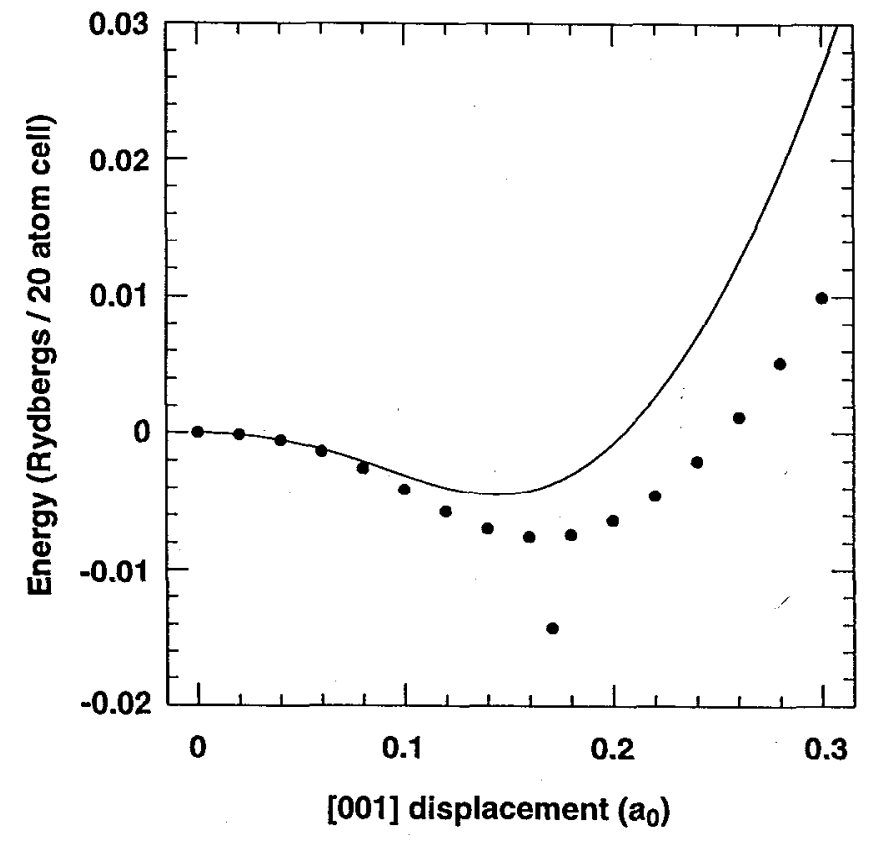

Figure 1: A plot of the change in grain boundary excess energy in the atomistic simulation as a function of the relative shift of the crystals along [001]. The line represents a rigid translation of one grain with respect to the other. The points represent calculations where the grain translations were held fixed and a relaxation allowed. The lowest point is the full $3 D$ relaxation of the structure. 
(a)

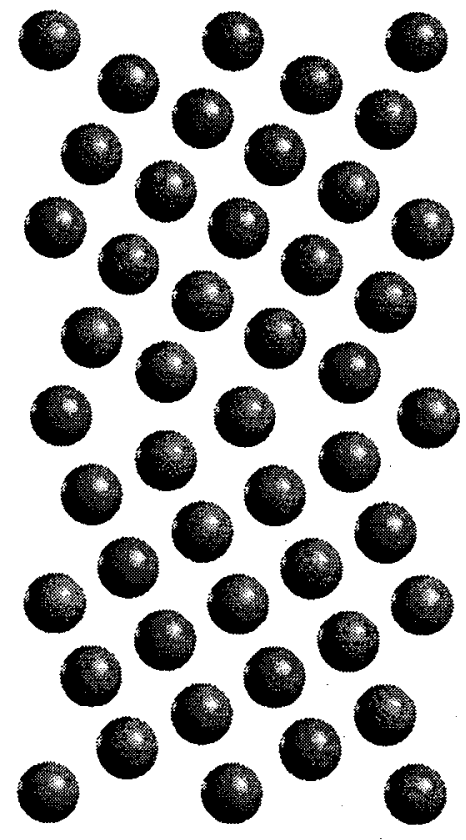

(b)

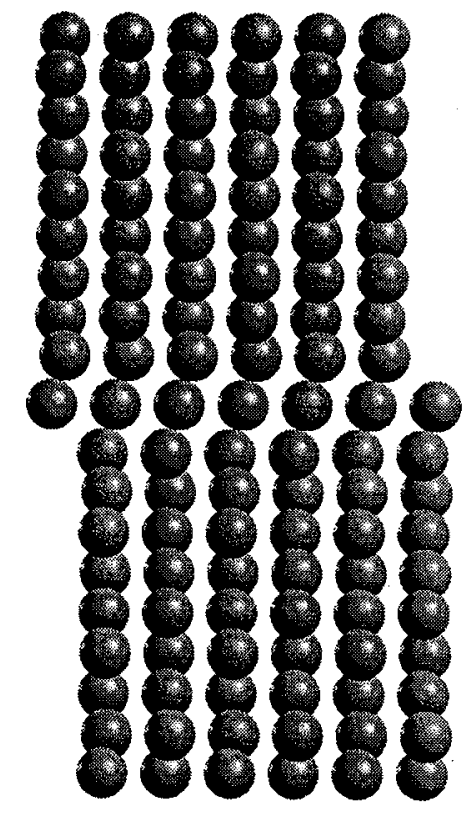

Figure 2: The predicted structure of the $\Sigma 5(310) /[001]$ STGB in Ta from atomistic simulations using interatomic potentials derived from MGPT. In (a) the structure is viewed along the common [001] tilt axisand in (b) it is viewed perpendicular to the tilt axis along [130].

The predicted structure for the $\Sigma 5(310) /[001]$ STGB in Ta is shown in Fig. 2(a) and (b). This boundary has the [001] crystal direction as the common tilt axis and Fig. 2(a) views the structure along this direction. A notable feature is seen in the view perpendicular to the tilt axis (Fig. 2(b)) in that the crystal planes are not perfectly aligned, rather there is a shift of one crystal with respect to the other.

\section{EXPERIMENT}

In order to experimentally investigate the structure of the grain boundary shown in Fig. 2, we fabricated it by diffusion bonding precisely oriented single crystals of high purity tantalum. The single crystal of Ta was grown at the Institute for Solid State Physics in Chernogolovka, Russia, from high purity stock material provided from Cabot Corp. The Ta was further purified by zone refining in high vacuum. The liquid zone was formed by electron beam heating from an annular electrode and five passes of the liquid zone were taken, with the last pass starting in a seed crystal oriented for growth along [310]. The crystal diameter was $18 \mathrm{~mm}$ with approximately $200 \mathrm{~mm}$ length.

Two $5 \mathrm{~mm}$ slices of the single crystal boule perpendicular to the growth axis were taken by electric discharge machining (EDM). The crystal slices were oriented with Laue backscatter $\mathrm{x}$-ray diffraction and lapped in specially designed fixtures to orient the faces to within $0.1^{\circ}$ of (310). The faces were polished with flat polishing techniques to achieve a flatness of less than $100 \mathrm{~nm}$ across the $15 \mathrm{~mm}$ diameter faces. Finally, the mutual orientation of the two slices was set by a $180^{\circ}$ rotation of one crystal with respect to the other around an axis perpendicular to the slice face. The process is fully described elsewhere [14].

The UHV Diffusion Bonding Machine has been described in detail elsewhere [15]. The Ta crystals where sputter cleaned immediately prior to bonding to remove the oxide layer that forms upon exposure to the atmosphere. Auger spectra taken of the surface confirmed the removal of the oxide. The crystals were bonded at $1500^{\circ} \mathrm{C}$ for $8 \mathrm{~h}$ under an applied load of $1 \mathrm{MPa}$. The bicrystal was further heat treated in a separate UHV apparatus at $2600^{\circ} \mathrm{C}$ for $48 \mathrm{~h}$ in order to eliminate any 


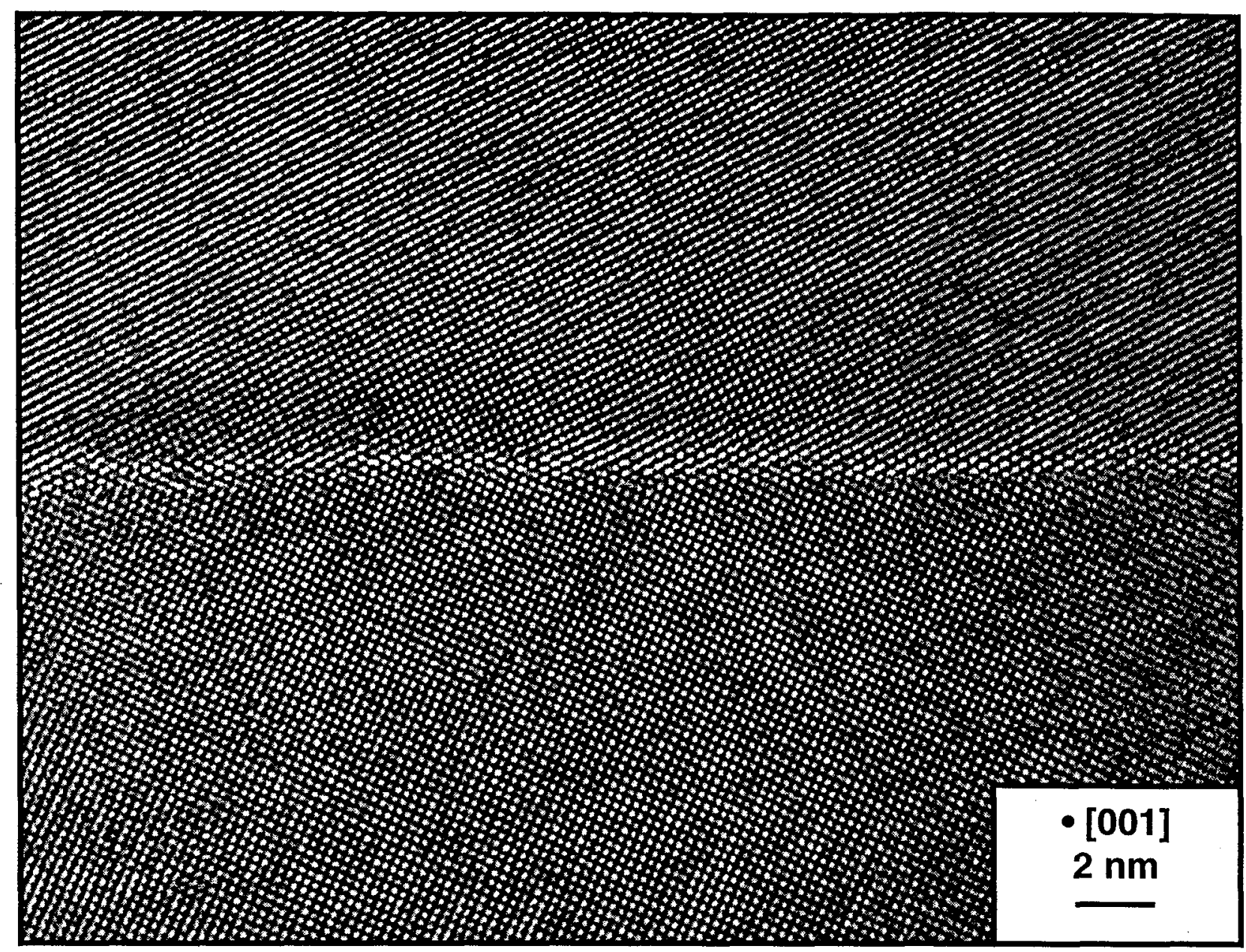

Figure 3: A high resolution electron micrograph of the $\Sigma 5$ (310)/[001] STGB in Ta with the direction of view parallel to the common [001] tilt axis. The direction is the same as in Fig. $2 a$.

residual porosity remaining at the grain boundary after the initial diffusion bonding step.

Two cylindrical cores were taken of the bicrystal by wire EDM cutting. The cores were $3 \mathrm{~mm}$ in diameter and contained the grain boundary along their axes. The two cylinders were cut with their axes along different directions: one along the common [001] direction and the other perpendicular to the first along the common [1 $\overline{3} 0$ ] direction. The cylinders were sliced to give $3 \mathrm{~mm}$ disks of $300 \mu \mathrm{m}$ thickness. The disks were gently lapped to $150 \mu \mathrm{m}$ thickness and $50 \mu \mathrm{m}$ deep dimples were ground centered over the grain boundary on both sides. The final $50 \mu \mathrm{m}$ was thinned by electropolishing with an electrolyte of $1 \%$ hydrofluoric acid, $5 \%$ sulfuric acid, $34 \%$ butoxyethanol, and $60 \%$ methanol at $-20^{\circ} \mathrm{C}$. The dimpling assisted in locating the perforation to intersect the grain boundary. Nonetheless, perforations seldom intersected the grain boundary. In these cases, the specimens were immersed in a solution of $60 \%$ sulfuric acid, $25 \%$ nitric acid, and $15 \%$ hydrofluoric acid that enlarged the specimen hole while retaining thin area until it intersected the grain boundary.

The high resolution imaging was performed on a Philips CM300FEG-ST. The images of the grain boundary structure along. [001] were acquired on film. The images along [1 $\overline{3} 0]$ were acquired on a $2 \mathrm{k} \times 2 \mathrm{k}$ CCD array after energy filtering for only zero loss electrons with a $20 \mathrm{eV}$ window on a Gatan Imaging Filter.

\section{RESULTS}

The high resolution image acquired parallel to the tilt axis is shown in Figure 3. The grain boundary displays a plane of mirror symmetry in this projection. To reveal the shift predicted by the atomistic simulations, an image perpendicular to the tilt axis is required. A high resolution micro- 

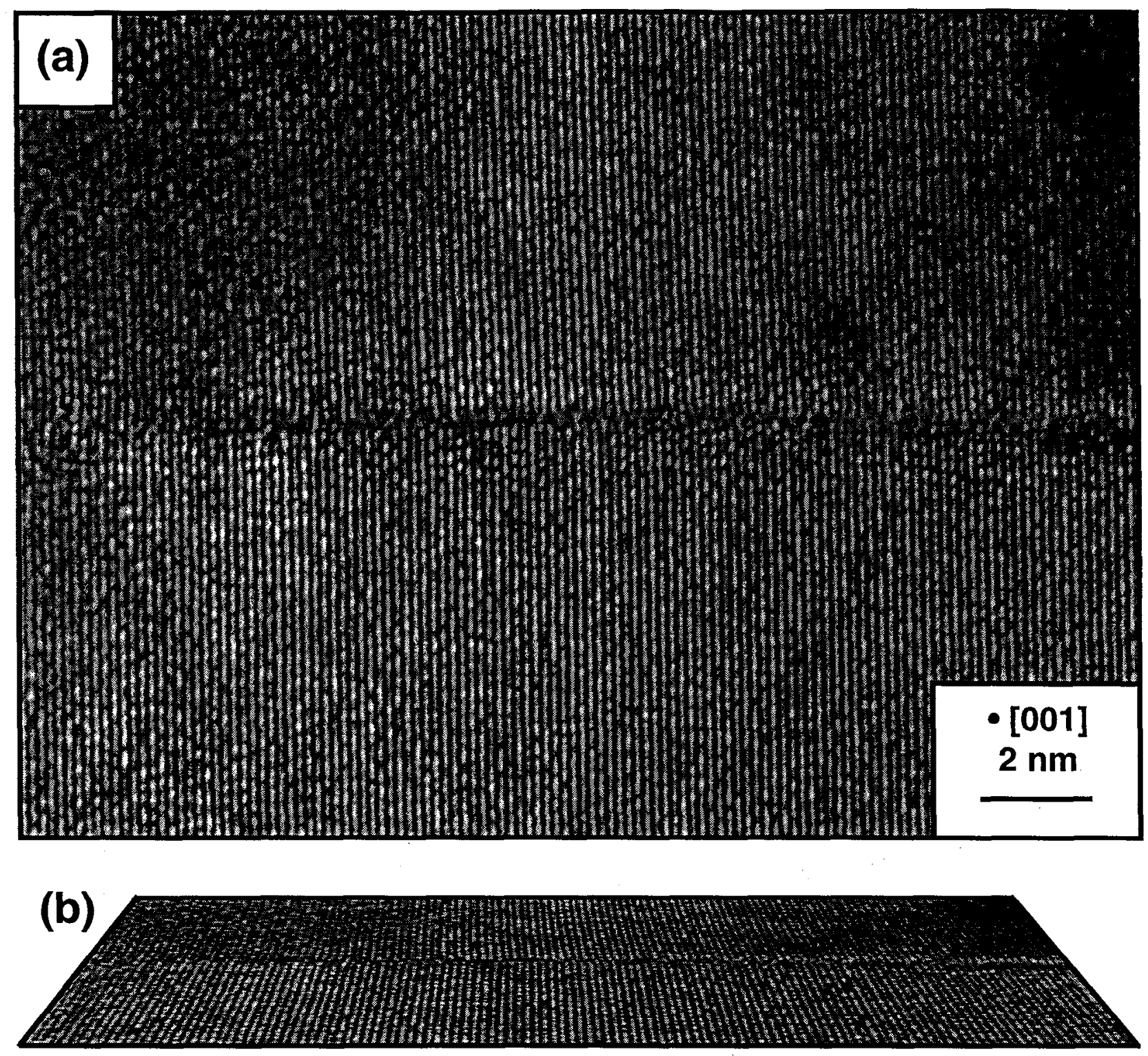

Figure 4: (a) A high resolution electron micrograph of the $\sum 5$ (310)/[001] STGB in Ta with the direction of view perpendicular to the common tilt axis. The direction is the same as in Fig. $2 b .(b)$ A glancing angle perspective view of the same image as shown in (a).

graph acquired in this direction is shown in Figure 4(a). Each crystal is imaged along [1 $1 \overline{3} 0]$. The two crystal planes with the largest interplanar spacing containing [1 $\overline{3} 0]$ are (002) and (310) at $1.65 \AA$ and $1.04 \AA$, respectively. The information limit for this microscope is approximately $1.2 \AA$. Thus only the (002) set of atomic planes are imaged as fringes in this image. The shift of the crystals along [001] is revealed by sighting down the (002) fringes in the image and noting their alignment as they cross the grain boundary. The shift is most easily seen in a glancing angle perspective view, such as that shown in Figure 4(b). The disregistry of the (002) planes as they cross the grain boundary is evident.

\section{DISCUSSION AND CONCLUSION}

The high resolution images of the $\Sigma 5(310) /[001]$ STGB in Ta show that the boundary contains a rigid body shift of the crystals along [001]. The shift is evident from a qualitative inspection of Fig. 4(a), assisted by the glancing angle perspective view shown in Fig. 4(b). The magnitude 
of the shift is approximately $1 / 4 \mathrm{a}_{0}$ which is comparable to the shift predicted by the atomistic simulations.

The results of the present study on Ta, when combined with previously reported results on $\mathrm{Nb}$ [8] and Mo [9], reaffirms the predictive power of the MGPT derived potentials for modeling defect structures in the $\mathrm{BCC}$ metals.

\section{ACKNOWLEDGEMENTS}

We would like to thank S.L. Weinland and R.A. Bliss for their assistance with specimen preparation. This work was performed under the auspices of the United States Department of Energy and the Lawrence Livermore National Laboratory under contract number W-7405-Eng-48.

\section{REFERENCES}

1. W. Xu and J. A. Moriarty, Phys. Rev. B, 54 [10] 6941-6951 (1996).

2. M. I. Baskes, Phys. Rev. B, 46 [5] 2727-2742 (1992).

3. S. M. Foiles, Phys. Rev. B, 48 [7] 4287-4298 (1993).

4. A. G. Marinopoulos, V. Vítek and A. E. Carlsson, Philos. Mag. A, 72 [5] 1311-1330 (1995).

5. J. A. Moriarty, Phys. Rev. B, 42 [3] 1609-1628 (1990).

6. D. G. Pettifor, M. Aoki, P. Gumbsch, A. P. Horsfield, D. Nguyen Manh and V. Vítek, Mat. Sci. Eng. A, 192/193 24-30 (1995).

7. A. E. Carlsson, Phys. Rev. B, 44 [13] 6590-6597 (1991).

8. G. H. Campbell, S. M. Foiles, P. Gumbsch, M. Rühle and W. E. King, Phys. Rev. Lett., 70 [4] 449-452 (1993).

9. G. H. Campbell, J. Belak and J. A. Moriarty, Acta Mater., in press (1999).

10. J. A. Moriarty, Phys. Rev. B, 38 [5] 3199-3231 (1988).

11. J. A. Moriarty, Phys. Rev. B, 49 [18] 12431-12445 (1994).

12. W. Bollmann, Crystal Defects and Crystalline Interfaces (Springer - Verlag, Berlin, 1970).

13. M. P. Allen and D. J. Tildesley, Computer Simulation of Liquids (Oxford University Press, New York, 1987).

14. W. L. Wien, G. H. Campbell and W. E. King in Microstructural Science, edited by D. W. Stevens, E. A. Clark, D. C. Zipperian and E. D. Albrecht (Microstructural Science 23, Materials Park, OH 1996) pp. 213-218 ;presented at 28th International Metallography Society Convention. 15. W. E. King, G. H. Campbell, A. W. Coombs, G. W. Johnson, B. E. Kelly, T. C. Reitz, S. L. Stoner, W. L. Wien and D. M. Wilson in Joining and Adhesion of Advanced Inorganic Materials, edited by A. H. Carim, D. S. Schwartz and R. S. Silberglitt (Mat. Res. Soc. Symp. Proc. 314, Pittsburgh, PA 1993) pp. 61-67. 